Conclusion The risk of AMR GC and the associated loss of convenient outpatient therapy is of great concern to the military medical community. Comparable data across geographically distinct regions is essential for monitoring AMR GC and implementing appropriate countermeasures in locations where service members are or could be deployed.

Disclosure No significant relationships.

\section{P041 HOW IS THE VALUE OF POINT-OF-CARE TESTS FOR STIS NEGOTIATED IN THE CONTEXT OF A NATIONALISED HEALTH SYSTEM?}

${ }^{1}$ Agata Pacho*, ${ }^{1}$ Emma Heming De-Allie, ${ }^{1}$ Martina Furegato, ${ }^{1}$ Emma Harding-Esch, ${ }^{2} \mathrm{~S}$ Tariq Sadiq, 'Sebastian Fuller. 'St George's, University of London, Applied Diagnostic Research and Evaluation Unit, Institute for Infection and Immunity, London, UK; ${ }^{2}$ St George's University of London, Applied Diagnostic Research and Evaluation Unit (ADREU), Institute for Infection and Immunity, London, UK

\subsection{6/sextrans-2019-sti.247}

Background Affordability, ease-of-use, rapid turnaround times and laboratory-equivalent accuracy have been identified as essential characteristics for point-of-care tests (POCTs) for STIs. Yet meeting these benchmarks does not guarantee POCT adoption into sexual health services (SHSs). Qualitative research can provide contextual understanding for how POCT characteristics are valued in relation to structural and political processes within health systems.

Methods We invited England SHSs interested in adopting POCTs for STIs to participate in the Facilitators to Adoption study, focused on understanding key facilitators and barriers to technology adoption within their services. Within these SHSs, we conducted in-depth interviews with key stakeholders selfidentified as integral to adoption of POCTs into their services. Interviews were thematically analysed in NVIVO 11 to examine 'appropriateness' and 'usefulness' of POCT characteristics in the context of participating SHSs and the overall priorities of the National Health Service (NHS) in England.

Results 31 healthcare professions from 6 SHSs were interviewed between April and November 2018. Interviewees identified cost-effectiveness and ease-of-use as important in assessing POCTs attractiveness to their services. POCTs were seen by service leads as cost saving only if they affect costs directly incurred by the service, while potential effectiveness of POCTs was assessed by clinicians in the context of their potential for improving appropriate and timely treatment and care to area-specific priority patient groups. In some SHSs, the potential for POCTs to be seen by commissioners as increasing the competitiveness of their service by meeting new policy targets was an important factor driving adoption.

Conclusion The need for POCTs and their desirable characteristics are negotiated within complex processes of funding constraints, service restructuring and political commitments to increasing inclusivity of care. Our findings suggest that service leaders may find areas to leverage adoption of POCTs by focusing on the tests' potential to increase service relevance and competitiveness.
Disclosure No significant relationships.

\section{P043 REGIONAL DIFFERENCES IN STI TESTING BARRIERS AMONG ONLINE TESTERS IN BRITISH COLUMBIA, CANADA}

${ }^{1}$ Aidan Ablona, ${ }^{1}$ Troy Grennan, ${ }^{1}$ Travis Salway, ${ }^{2}$ Jean Shoveller, ${ }^{3}$ Christopher Fairley, ${ }^{4}$ Mel Krajden, ${ }^{5}$ Maja Karlsson, ${ }^{5}$ Lorena Hiscoe, ${ }^{6}$ Sophie Bannar-Martin, ${ }^{6}$ Dee Hoyano, 'Oralia Gomez-Ramirez, ${ }^{1} \mathrm{H}$ siu-Ju Chang, ${ }^{2}$ Kimberly Thomson, ${ }^{1}$ Devon Haag, ${ }^{1}$ Mark Gilbert*. 'BC Centre for Disease Control, Clinical Prevention Services, Vancouver, Canada; ${ }^{2}$ University of British Columbia, School of Population and Public Health, Vancouver, Canada; ${ }^{3}$ Monash University, Central Clinical School, Carlton, Australia; ${ }^{4} B C$ Centre for Disease Control, Public Health Laboratory, Vancouver, Canada; Interior Health Authority, Kelowna, Canada; ${ }^{6}$ sland Health Authority, Victoria, Canada

\subsection{6/sextrans-2019-sti.248}

Background GetCheckedOnline (GCO), an online sexuallytransmitted infection (STI) testing service in British Columbia, launched in Vancouver, then expanded to two health regions (Island and Interior), including smaller urban and rural communities. We hypothesized that barriers to STI testing among GCO clients would be greater outside of Vancouver, due to a lower availability of existing STI services regionally.

Methods In 2015-2018, GCO clients were invited to participate in an online survey about STI testing barriers and facilitators at individual (e.g., embarrassment), healthcare provider (e.g., comfort discussing sexual health), clinic (e.g., distance, hours), and social levels (e.g., peer norms). We conducted Chi-squared, Fisher's exact, and t-tests for bivariate analyses (Vancouver vs. Interior, Vancouver vs. Island); significant results $(\mathrm{p}<0.01)$ are shown.

Results 583 GCO clients completed surveys: 299 (51\%) Vancouver, 203 (35\%) Island, and 81 (14\%) Interior. Vancouver respondents included proportionately more men who have sex with men, racialized minorities, and immigrants. A higher proportion of Interior (24\%) and Island respondents (18\%) reported testing for the first time compared to Vancouver $(8 \%)$. More Vancouver respondents reported testing through GCO for routine testing (possible other reasons: symptoms, new relationship). We found no regional differences in other barriers at individual or provider levels. Fewer Island respondents reported delaying testing in the past year due to access issues compared to Vancouver respondents (57\% vs 69\%), which was not explained by differences in testing history. At a social level, fewer Interior respondents reported regular STI testing as a peer norm (31\% vs 58\% Vancouver).

Conclusion Our findings suggest that testing barriers generally may be more universal than region-specific among users of an online STI testing service. Moreover, despite the apparently wider availability of in-person sexual health services in Vancouver, barriers in accessing these services may persist. Future socio-demographic analyses and additional research (e.g., community surveys) may help to contextualize these findings.

Disclosure No significant relationships. 